\section{Gesundheitsministerium fördert Allergieprojekte}

\section{Mit bisher etwa 60 Millionen DM förderte die Bundesregierung im Rahmen ihres Programms „Gesundheitsforschung 2000" seit 1987 die Allergie- und Lungenforschung. Für wichtig hält sie außerdem die Prävention und gesundheitliche Aufklärung der Patienten.}

Im Rahmen der Allergieforschungsprojekte will das Bundesministerium für Gesundheit vorrangig die Struktur für die klinische Forschung verbessern, Kliniker und Grundlagendisziplinen sollen stärker zusammenarbeiten. Ziel ist außerdem, eine forschungsorientierte Organisation der Kliniken zu schaffen, lokale Forschungsschwerpunkte $\mathrm{zu}$ bilden und erfolgreiche Forschergruppen langfristig in die beteiligten Kliniken und Institute zu integrieren.

Darüber hinaus werden internationale Allergieforschungsprojekte aus Mitteln der europäischen Kommission finanziert. So sieht das fünfte Rahmenprogramm der Europäischen Gemeinschaft im Bereich Forschung, technologische Entwicklung und Demonstration (1998-2002), das in Kürze verabschiedet wird, Forschungsmaßnahmen im Bereich Umwelt und Allergien vor.

\footnotetext{
Vorsorge und Früherkennung

Da bei Erkrankungen des allergischen Formenkreises der Prävention und der damit verbundenen gesundheitlichen Aufklärung eine wesentliche Bedeutung zukommt, ist dieser Arbeitsbereich zudem Bestandteil des Ressortforschungsprogramms des Bundesministeriums für Gesundheit. Um Versorgung, Vorsorge, Früherkennung und Aufklärung allergiekranker Kinder und Jugendlicher $\mathrm{zu}$ verbessern, werden in verschiedenen Modellprojekten neue Lösungsstrategien erprobt.

Im Versorgungsbereich werden allergiekranke Kinder und ihre Familien bei der Bewältigung ihrer Erkrankung unterstützt. Ein aktuelles Projekt fördert die Konsensfindung und Qua-
}

litätssicherung im Bereich der Neurodermitis-Schulung.

\section{Im Zentrum: die DISA}

Zentrale Bedeutung für die gesundheitliche Aufklärung hat das Projekt „Dokumentations- und Informationsstelle für Allergiefragen im Kindesalter" (DISA). Mit der DISA sind Kooperationspartner zu einem dynamischen Informationssystem vernetzt. Die geschlossene Kommunikationsstruktur (,Wissenschaftliches Forum“) soll den Wissensaustausch und die Konsensbildung mit Experten und Wissenschaftlern ermöglichen und er- leichtern. Eingebunden sind darin Selbsthilfeorganisationen und die Bundeszentrale für gesundheitliche Aufklärung (BzgA). Ärzten, Krankenkassen, der Bevölkerung und den Betroffenen sollen wissenschaftlich abgesicherte Allergie-Informationen verfügbar gemacht werden.

Gedacht war die DISA nicht als unmittelbarer Ansprechpartner für Patienten oder deren Familien, sondern sollte durch die optimale Information von Behörden, behandelnden Ärzten, anderen im Gesundheitswesen Tätigen und Selbsthilfeorganisationen indirekt für Patienten und Betroffene hilfreich wirken. Die aktivsten und mitgliedstärksten Selbsthilfeorganisationen Deutscher Allergie- und Asthmabund e.V. (DAAB) und die Arbeitsgemeinschaft Allergiekrankes Kind (AAK) sind bereits Kooperationspartner der DISA.

(red)

Quelle: Dr. Albert Statz: „Netzwerkprojekte des BMG im Bereich Allergie-Prävention“. 25. Kongreß des Ärzteverbandes Deutscher Allergologen, Göttingen 1998.

\section{Selbsthilfegruppen kooperieren}

\section{Das Netzwerkprojekt DISA}

Die Akademie für Kinderheilkunde und Jugendmedizin e.V. ist 1996 vom Bundesministerium in einem Modellprojekt mit dem Aufbau einer „Dokumentations- und Informationsstelle für Allergiefragen im Kindesalter" beauftragt worden. Sie hat die praktische Durchführung des Projektes an Frau Dr. Sabine Schmidt, Prof. K. E. v. Mühlendahl und die allergologische Abteilung des Kinderhospitals Osnabrück übertragen und ihre Arbeit im Mai 1996 in Osnabrück aufgenommen.

Die Aufgaben der DISA sind:

- den jeweiligen wissenschaftlichen Stand zu allergologischen Themen im Kindes- und Jugendalter zu erfassen, zusammenzufassen und ggf. kommentiert für interessierte Benutzergruppen verfügbar zu machen. Der jeweilige „Stand der Wissenschaft“" soll durch die Meinungen und Urteile von Experten geformt werden,

- Anfragen schnell und kompetent zu beantworten,

- mit bestehenden Informationsangeboten von pädiatrischen Fachgesellschaften und Selbsthilfeorganisationen zu kooperieren,

- durch ihre vielfältigen Kontakte mit anderen Informationsanbietern Auskunftssuchende schnell an kompetente und hilfreiche Angebote weiter zu verweisen.

Ansprechpartner: Dr. Sabine Schmidt, DISA, Iburger Str. 200, D-49082 Osnabrück, Tel.: (05 41) 5848621 , Fax: (05 41) 5848622. 